\title{
MULTIWAVELENGTH LIDAR OBSERVATION OF THE ATMOSPHERIC RESPONSE TO THE 20TH MARCH 2015 PARTIAL SOLAR ECLIPSE IN ROME TOR VERGATA: PRELIMINARY RESULTS.

\author{
Gian Luigi Liberti ${ }^{1}$, Davide Dionisi ${ }^{1}$, Stefano Federico ${ }^{1}$, Fernando Congeduti ${ }^{1}$ \\ Italy,*Email: g.liberti@isac.cnr.it
} \\ ${ }^{1}$ Institute of Atmospheric Sciences and Climate-CNR, Via del Fosso del Cavaliere 100, 00133 Roma,
}

\begin{abstract}
This study reports some preliminary analyses of multichannel lidar measurements taken in Rome Tor Vergata (Italy) during the 20th March 2015 partial solar eclipse. The objective is assessing the capability of the instrument to document the effect of the eclipse in the lower troposphere, with a particular emphasis on the information content at relatively small temporal and spatial scales.
\end{abstract}

\section{INTRODUCTION}

Solar eclipses are a unique opportunity for studying the atmospheric response to the large scale and sharply variation of the incoming solar radiation. Due to the rarity of the event, only few studies by the means of lidar have been conducted to characterize the phenomenon. One of the main observed effects is on the formation and the evolution of the Planetary Boundary Layer (PBL) during the solar eclipse. An aerosol backscatter lidar was used by Kolev et al. [1] to investigate the dynamics of the PBL during the solar eclipse of 11 August 1999, over Bulgaria. This study demonstrated that the solar eclipse affects the meteorological parameters of the atmosphere near the ground, the ozone concentration and the height of the mixing layer. During the solar eclipse of 29 March 2006, lidar data from two sites were used to characterize the Planetary Boundary Layer (PBL) height evolution over Greece, during the solar eclipse of 29 March 2006 [2]. In this case the solar eclipse induced a decrease of the PBL height, indicating a suppression of turbulence activity similar to that during the sunset hours.

According with astronomic data the eclipse in Rome started at about 8:24 UTC and ended at 10:43 UTC reaching the maximum (53\% totality) at about 9:32 UTC. The aim of this paper is to document its effect in the troposphere through the multi-wavelength Rayleigh-Mie-Raman lidar located at Rome Tor Vergata (Italy). The analysis of the atmospheric response at different temporal and spatial scales has been performed.

\section{METHODS}

\subsection{METEOROLOGICAL DATA}

Figure 1 shows 1-minute sampled surface meteorological data from the automatic weather station on the site of the lidar system. As expected during the eclipse there is a cooling of the air temperature caused by the decrease of the solar short-wave irradiance. The perturbation to the solar irradiance caused by the eclipse is larger than $300 \mathrm{~W} / \mathrm{m}^{2}$, while the surface temperature decrease is greater than $1^{\circ} \mathrm{C}$. There is a time shift between the effective air temperature estimated by the downward LW radiation at the air temperature at the surface during the eclipse (the minimum of the latter lasts that of the former). This difference is likely caused by the different heat capacity of the two systems, the air temperature at the surface being influenced by the ground temperature. The maximum of the pressure is partially due to the semidiurnal tides. The $2 \mathrm{~m}$ air density was estimated from pressure and temperature. The air density shows an increase at the surface caused by the stratification of the atmosphere during the eclipse. It is also important to note that the curve of the solar irradiance, if we exclude the perturbation of the eclipse, shows the typical behavior of a clear sky day. Also, the surface winds are small and their evolution shows an increase during the afternoon, caused by the intensification of the sea-breeze and valleymountains flow. Thus, with the exception of the eclipse perturbation, the evolution of the PBL follows that of a clear sky wind calm evolution.

\subsection{DESCRIPTION OF THE LIDAR SYSTEM AND DATA PROCESSING}

The Rayleigh-Mie-Raman (RMR) lidar of ISAC CNR is part of the experimental site in the 
suburban hilly area of Tor Vergata $\left(41.88^{\circ} \mathrm{N}\right.$, $\left.12.68^{\circ} \mathrm{E}, 107 \mathrm{~m} \mathrm{ASL}\right)$.
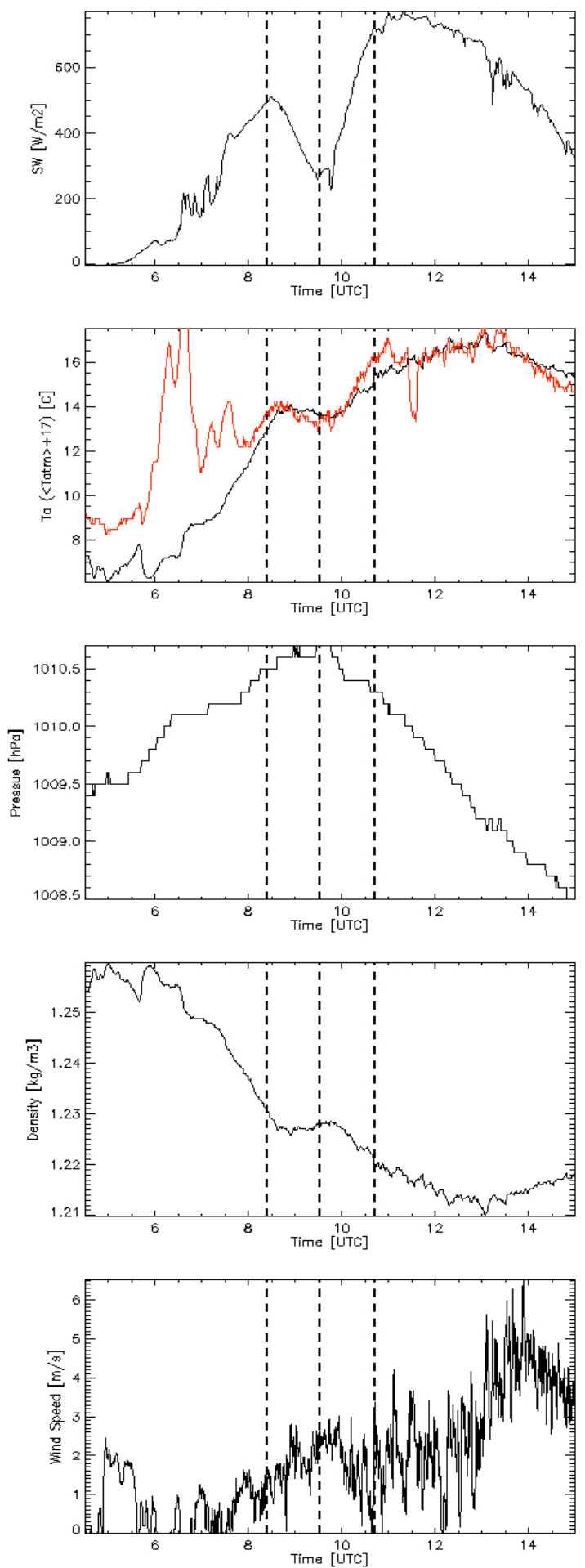

Figure 1. $2 \mathrm{~m}$ meteorological data: Red line in the air temperature panel is the effective sky temperature $(<\mathrm{Tatm}>)$ as estimated from the downward LW radiation (to match the $2 \mathrm{~m}$ air temperature range the plotted line is $<$ Tatm $\left.>+17^{\circ} \mathrm{C}\right)$.

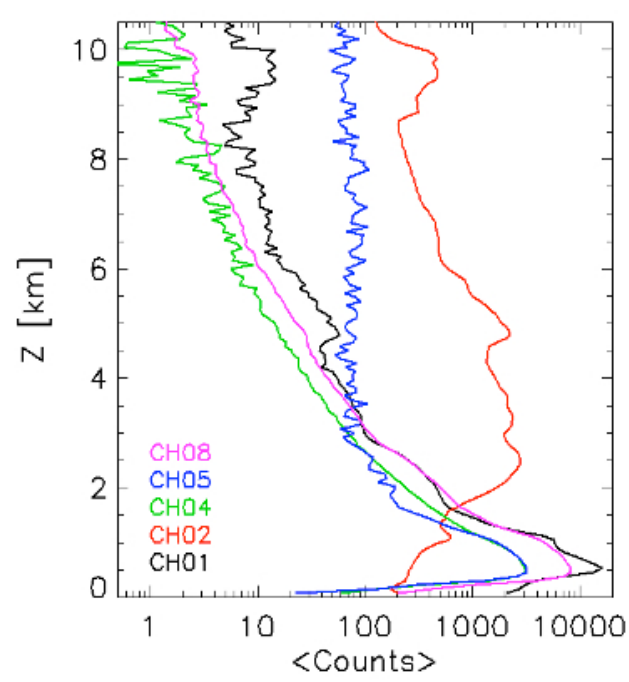

Figure 2. Mean profiles of dead-time corrected counts.

The transmitter is based on an Nd:YAG laser with 2nd $(532 \mathrm{~nm})$ and $3 \mathrm{rd}(355 \mathrm{~nm})$ harmonic generators. Backscattered radiation is collected and analyzed at 4 wavelengths of interest: 355 and $532 \mathrm{~nm}$ for the elastic backscatter and 386.7 and $407.5 \mathrm{~nm}$ for Raman scattering of $\mathrm{N}_{2}$ and $\mathrm{H}_{2} \mathrm{O}$ molecules, respectively. The receiver is in a multiple-telescope configuration [3], allowing the sensing of a wide-altitude atmospheric interval. For the Raman and elastic backscatter, two different collection channels $(\mathrm{CHs})$ are employed: one for the lower range $(0.1-5-\mathrm{km}$ altitude, using a $30-\mathrm{cm}$ telescope and an optical fiber) and one for the upper range (2-13-km altitude, using an array of nine $50-\mathrm{cm}$ aperture telescopes and an optical fiber bundle). An additional $15-\mathrm{cm}$ telescope is used to collect the $532 \mathrm{~nm}$ elastic backscatter from the lower atmosphere (0.5-8 $\mathrm{km})$. During the eclipse, the following signals were acquired with the $30 \mathrm{~cm}$ telescope: the 532 and $355 \mathrm{~nm}$ elastic backscattering ( $\mathrm{CHO2}, \mathrm{CH} 08)$; the N2 (CH04 at $387 \mathrm{~nm})$ and $\mathrm{WV}(\mathrm{CH} 05$ at 407 $\mathrm{nm})$ Raman backscatter. The $532 \mathrm{~nm}$ elastic backscattering was also acquired with the $15 \mathrm{~cm}$ telescope (CH01). In the following, we analyze the signals corrected for the background value and the dead-time. During daytime the background is a function of the intensity of solar backscattered radiation and therefore should vary consistently during the eclipse. 

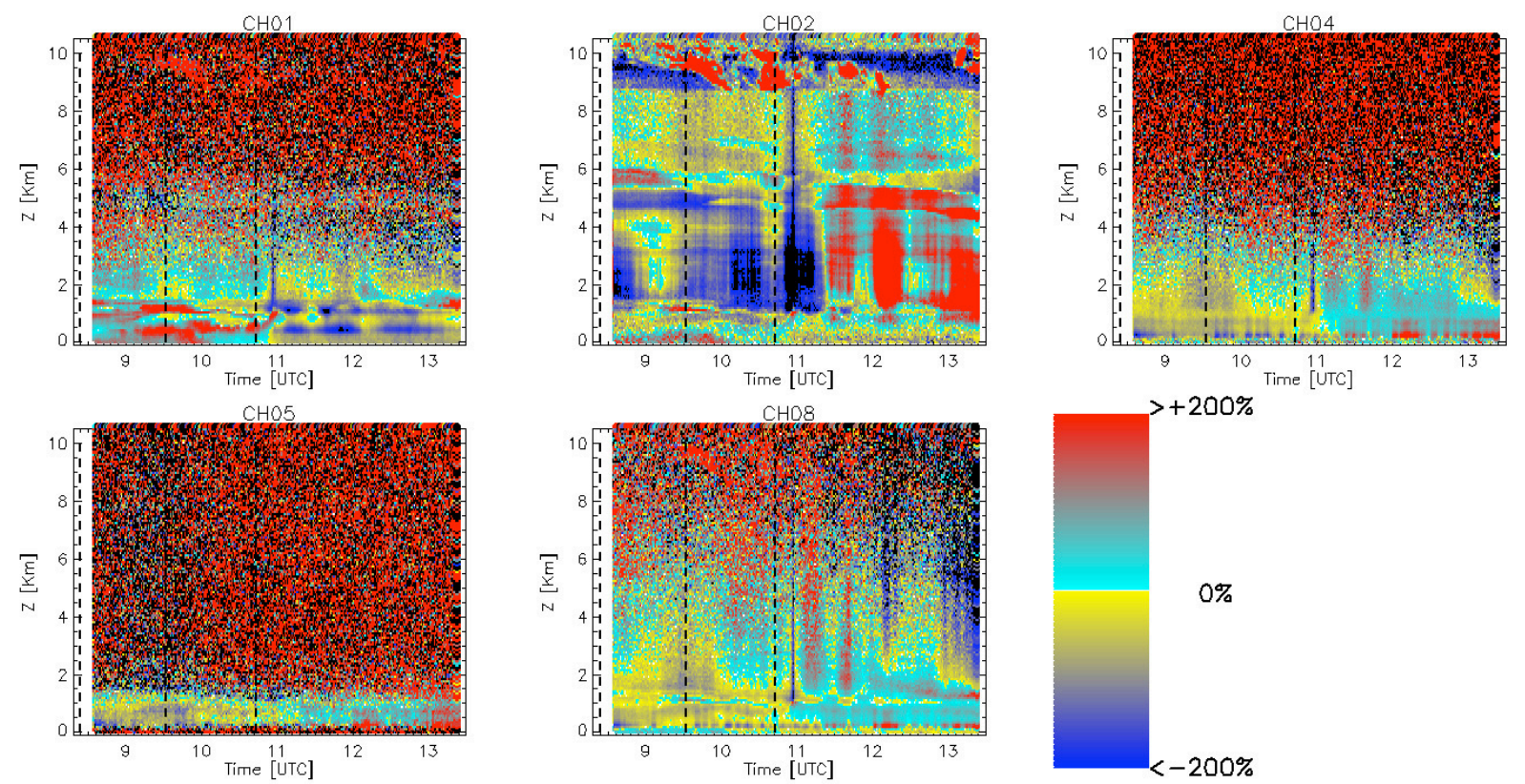

Figure 3. Time series of the profiles of the anomalies of the signals [\%], with respect to the average (Fig.2). Dashed vertical bars are plotted in correspondence of beginning, maximum and ending time of the eclipse.
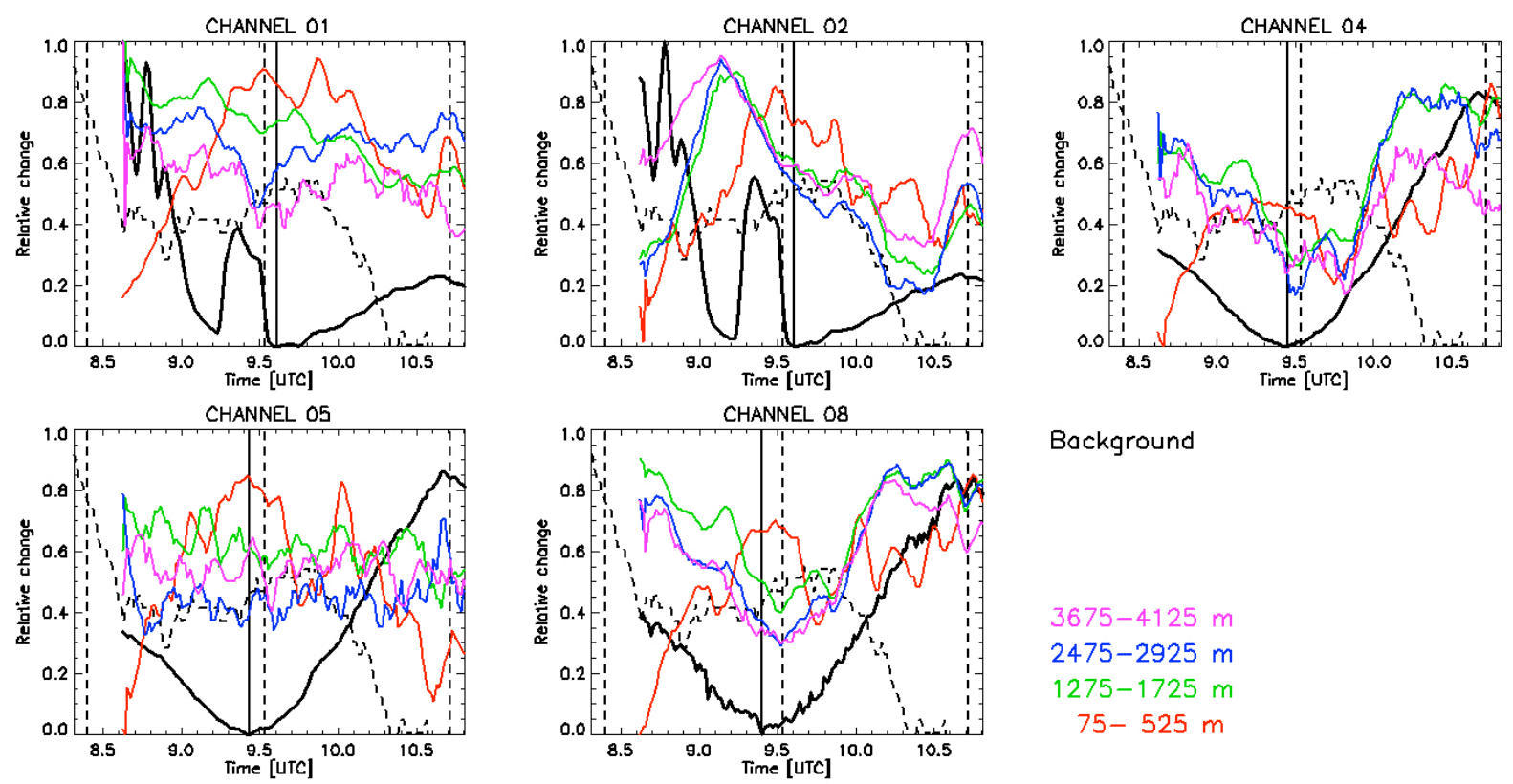

Bockground

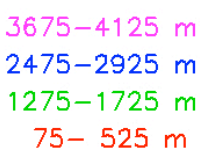

Figure 4. Time series of corrected signals for different atmospheric layers averaged over a 7 ' by $450 \mathrm{~m}$ moving box and normalized between 0 and 1 during the eclipse (dashed vertical bars in correspondence of beginning, maximum and ending time of the eclipse). Also plotted the normalized background value (black line) and the time of its minimum (black continuous vertical line).

\section{RESULTS}

Figure 2 shows the mean profiles of the dead-time corrected counts acquired by the different channels between 8:38-13:28 UTC. During the eclipse, the main permanent features on the signals are the presence of the aerosols in the PBL up to $2 \mathrm{~km}$ and a cirrus between 8.7 and $10 \mathrm{~km}$.
Figure 3 shows the time series of single 1-min profiles during the same time window represented as anomaly [\%] with respected to the averaged profiles. Channels 4 and 8 are the most sensitive to the atmospheric density. Considering the anomaly of the signals (Figure 3), during the eclipse it is apparent a decrease of the density 
between $500 \mathrm{~m}$ and $2000 \mathrm{~m}$. This feature is clear also in Figure 4, which shows the relative change of the signals averaged for different atmospheric layers. However, for channels 4 and 8, in addition to the decrease of the signal for the levels above $525 \mathrm{~m}$, there is an increase of the signals for the layer between $75 \mathrm{~m}$ and $525 \mathrm{~m}$, especially during the first part of the eclipse. This behavior shows the stratification of the atmosphere during the eclipse, due to the decrease of the solar irradiance. After the eclipse, the PBL shows the behavior of a clear sky, calm-wind day with the increase of the density between $500 \mathrm{~m}$ and 2-3 $\mathrm{km}$ (above those levels the Lidar signal becomes noisy) and a decrease at the lowest levels, as a consequence of the mixing of the PBL.

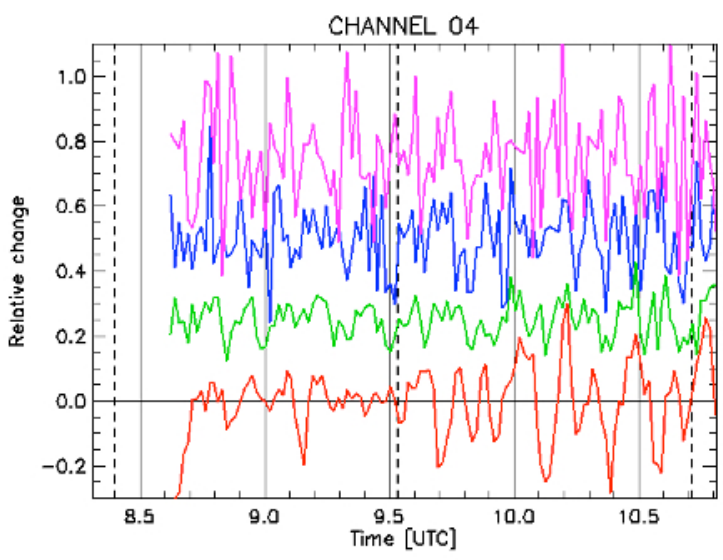

Figure.5 Time series of vertically averaged 1-min anomalies (with respect to the time averaged values Fig.4) for $\mathrm{CHO} 4$ at different atmospheric levels. Same legend as Fig.4 for the altitude of the level. Dashed vertical lines are the beginning, the maximum and the ending time of the eclipse.

Figure 5 shows the time series of vertically averaged 1-min anomalies (with respect to the time averaged values Fig.4) for $\mathrm{CHO} 4$ at different atmospheric levels. The anomalies seem to show some periodic behavior. Assuming that within the acquisition cycle of a single laser shot there are no changes of periodic nature in the performances of the whole acquisition chain, a periodic behavior in the signal could be generated by a periodic variation of the power of the emitting laser as well as of the properties of the atmosphere. The latters can modulate the measured signal in two ways: through the variability of the signal extinction due to the atmosphere below the backscattering level, through the variability of the properties of the backscattering level. With the above assumption, a periodicity in the signal due uniquely to a possible variation of the laser power should appear with the same frequency at all levels. Because of the different behavior with the altitude we may conclude that the observed fluctuations with period between $5^{\prime}$ and $10^{\prime}$ are due to changes in the atmospheric properties. Figure 5 shows also a decrease of the amplitude of the gravity waves, whose characteristics differs at different levels. This damping could be caused by the decrease of the surface heat forcing and we are currently investigating this issue.

\section{CONCLUSIONS}

During the eclipse the system observed an increase of the stratification of the atmosphere. The delay in the atmospheric response, of few minutes is consistent with what observed at the surface with the 2-min meteorological data. The occurrence of high frequency (period less than about $10 \mathrm{~min}$ ) variations has been shown. From this preliminary analyses we exclude that the observed high frequency variability is due uniquely to instrument induced periodic signals. From a very qualitative analysis it seems that the altitude of the base of the Cirrus observed in $\mathrm{CH} 2$ data during the whole morning could be correlated to the occurrence of the eclipse. Further investigations need to be conducted on this point. Following this signal-based preliminary analysis we plan to perform the study of the corresponding retrieved geophysical parameters and to compare with other available observations during the event.

\section{REFERENCES}

[1] Kolev, N., et al., 2005: Aerosol Lidar and in situ ozone observations of the planetary boundary layer over Bulgaria during the solar eclipse of 11 August 1999, Int. J. Remote Sens., 26, 3567-3584.

[2] Amiridis, V et al., 2007. Aerosol Lidar observations and model calculations of the Planetary Boundary Layer evolution over Greece, during the March 2006 Total Solar Eclipse. Atmos. Chem. Phys., 7, 6181-6189.

[3] Congeduti, F., et al., 1999: The multiplemirror lidar '9-eyes.' J. Opt., 1A, 185-191. 